\title{
Diacronie
}

Studi di Storia Contemporanea

$\mathrm{N}^{\circ} 15,3 \mid 2013$

Spazi, percorsi e memorie

\section{Il mare per comunicare (B)}

Sala B

\section{Stefania Elena Carnemolla}

\section{(2) OpenEdition \\ Journals}

\section{Edizione digitale}

URL: http://journals.openedition.org/diacronie/410

DOI: 10.4000/diacronie.410

ISSN: 2038-0925

Editore

Association culturelle Diacronie

Notizia bibliografica digitale

Stefania Elena Carnemolla, « II mare per comunicare (B) », Diacronie [Online], № 15, 3 | 2013,

documento 1, Messo online il 01 octobre 2013, consultato il 03 mai 2019. URL : http://

journals.openedition.org/diacronie/410; DOI : 10.4000/diacronie.410 


\title{
Diacronie
}

\section{1/}

\section{Il mare per comunicare}

\author{
Stefania Elena CARNEMOLLA*
}

Con un documento inedito di Ernesto DEL GRANDE

Nel 1885 il governo italiano stipulò un contratto con la Pirelli per la costruzione e la manutenzione di dodici cavi per l'allacciamento di varie isole con la terraferma. Come conseguenza dell'accordo, la Pirelli costruì a San Bartolomeo, vicino La Spezia, uno stabilimento per il rivestimento dei cavi, ordinando al cantiere Thompson la nave cablografica Città di Milano, che operò anche oltre i confini nazionali, naufragando nel 1919 a Filicudi. Nel 1944 Ernesto Del Grande, cavista Pirelli, scrisse di questa storia, organizzando anche un portfolio con settantandue immagini. Le due fonti, rispettivamente dell'Archivio Storico Pirelli e della Biblioteca Sormani, vengono ora pubblicate per la prima volta, con una selezione di trentasei immagini di quest'ultimo.

\section{Prefazione}

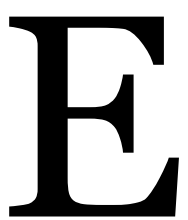

gr. Sig. Alberto PIRELLI, ricordando il suo imbarco sulla vecchia Città di Milano ritengo doveroso, da parte mia, d’inviarle copia di una mia relazione riguardante la storia di un simpatico ramo delle Industrie Pirelli cui dedicai con passione gli anni miei migliori e di cui sento tuttora viva la nostalgia, ed alla quale il suo venerato Papà era particolarmente attaccato. Questa relazione, da me scritta con altra destinazione, è stata ritenuta interessante dal Sig. Ing. Emanueli, che, perchè non andasse dispersa, dispose facesse parte delle raccolte delle Relazioni ufficiali del Dipartimento A. Distinti saluti, suo dev. ${ }^{\text {mo }}$ Ernesto Del Grande.

La lettera, datata Milano 19 aprile 1944, scritta su carta intestata della Società Italiana Pirelli accompagna un dattiloscritto originale del gennaio 1944, con correzioni autografe, di venticinque fogli e in sei capitoli di Ernesto Del Grande, cavista Pirelli. Il 
documento - titolo I cavi telegrafici sottomarini. Contributo alla storia delle Industrie Pirelli (ricordi di un vecchio cavista) - fa parte con la segnatura 1177 delle collezioni dell'Archivio Storico Pirelli di Milano, dove si conserva con la segnatura 2179 un esemplare doppione.

Nulla si sa di Ernesto Del Grande, se non che si salvò nel naufragio del piroscafo Città di Milano nel 1919 a Filicudi e che fu tra coloro che parteciparono a molte delle campagne della nave posacavi fatta costruire da Giovan Battista Pirelli nei cantieri Thompson di Sunderland.

Nella relazione si parla della nascita dell'industria italiana dei cavi telegrafici sottomarini, delle missioni del piroscafo Città di Milano nel Mediterraneo, Mar Rosso e Oceano Indiano, durante il terremoto di Messina, la guerra italo-turca, allorquando fu incaricato di tagliare i cavi nemici, la Grande Guerra, quindi del suo naufragio ${ }^{1}$ e della posa e riparazione dei cavi sottomarini. Né mancano riferimenti alla Cambria, la nave inglese noleggiata dalla Pirelli in ausilio al piroscafo Città di Milano durante la posa del cavo Siracusa-Bengasi. Così come non mancano immagini con il piroscafo Città di Milano mentre pesca un cavo da riparare, e tutte le altre, quelle con i grappini, il dinamometro, la puleggia di poppa, il gabinetto elettrico di bordo, la macchina di posa, l'indicatore d'imbando, le operazioni di salpaggio, i vari tipi di cavi, le boe.

$\mathrm{Si}$ vede in alcune di queste immagini un personaggio in abiti borghesi, lo stesso Ernesto Del Grande, e che ritorna in molte delle settantadue immagini di un album, in realtà un raccoglitore Pirelli, sulle campagne del piroscafo Città di Milano, della Cambria, del piroscafo Città di Milano II, già Grossherzog von Holdenburg, e oggi con la collocazione U Cons 49 delle collezioni della Biblioteca Sormani di Milano dove giunse con gli ex libris del Museo Navale cittadino nel dicembre del 1947.

Osservando le immagini, racconto di un'esperienza personale a bordo di navi posacavi, non potemmo che constatare, quando ce ne fu data notizia del tutto casualmente, come quel portfolio altro non fosse che di Ernesto Del Grande, più volte ritratto nell'album. Un documento prezioso per le notizie sulle campagne delle navi posacavi che operarono per la Pirelli, nonché per la storia della scienza e della tecnica.

\footnotetext{
${ }^{1}$ Nell'Archivio Storico Pirelli esistono sul naufragio del piroscafo Città di Milano altri due documenti di Ernesto Del Grande. Il primo del 18 giugno 1919 o Relazione sul naufragio della R.N. Città di Milano di quattro fogli dattiloscritti, con in disegno l'isola di Filicudi, la rotta della nave, il punto del naufragio e destinato alla Pirelli, fu redatto a Messina. Il secondo del 9 luglio 1919 o Relazione del Sig. Del Grande Ernesto sul naufragio della R.N. Città di Milano avvenuto nelle acque di Filicuri (Isole Eolie) il 16 giugno 1919 ore 13.30 circa, durante la Campagna N. 73 per Cavi Telegrafici Sottomarini di cinque fogli dattiloscritti, così come il primo con in disegno l'isola di Filicudi, la rotta della nave e il punto del naufragio, fu redatto a Milano. Il documento è interessante nella misura in cui trasmette i nomi del personale imbarcato, fornendo sul sinistro notizie ancora più dettagliate.
} 
Un racconto per immagini, accompagnato da altre dell'Archivio Storico Pirelli, che qui pubblichiamo benché parzialmente ${ }^{2}$, mentre integralmente viene ora pubblicata e per la prima volta la memoria di Ernesto Del Grande, il cavista Pirelli protagonista e testimone di una delle industrie più fiorenti dell'Italia che guardò al mare come progresso.

Stefania Elena Carnemolla

$\overline{\text { Ernesto DEL GRANDE, I cavi telegrafici sottomarini. Contributo }}$ alla storia delle Industrie Pirelli (Ricordi di un vecchio cavista)

Edizione a cura di Stefania Elena CARNEMOLLA

\section{Inizio dell'industria italiana dei cavi telegrafici sottomarini}

La fabbrica, la posa e l'esercizio di reti telegrafiche sottomarine, dopo il primo successo della posa di un cavo avvenuto felicemente nel 1866 tra l'Inghilterra e l'America del Nord, dopo vari drammatici tentativi da parte di una società inglese, era quasi un monopolio di società inglesi, che andavano gradatamente formando, in tutti i mari, Mediterraneo compreso, una vasta rete.

Le prime comunicazioni tra la penisola italiana e le isole maggiori e con l'Albania furono appunto eseguite da una società inglese che ne aveva anche la manutenzione.

Nel 1884 l'Ing. Giovan Battista Pirelli concepì l'idea audace di sostituirsi anche in questo campo all'industria straniera. Ma per fare ciò occorreva una attrezzatura che era difficile improvvisare. Lo Stabilimento di Milano, che già costruiva conduttori elettrici, non poteva servire che a fabbricare le anime isolate in guttaperca; per l'armatura dei cavi, in lunghe pezzature di centinaia di chilometri, occorreva invece uno stabilimento in riva al mare, onde poter caricarli sulla nave posacavi; e occorreva anche una di quelle navi speciali.

Iniziate le trattative con l'Amministrazione dei Telegrafi italiani, dopo un periodo di passione per l'Ing. Pirelli che vedeva le difficoltà e i rischi cui andava incontro assumendosi un impegno così complesso, nel novembre 1885 firmava a Roma due

${ }_{2}^{2}$ Pubblichiamo, secondo quanto concessoci dalla Direzione della Biblioteca Sormani, trentasei dei settandue fotogrammi dell'album, avendo avuto cura di selezionare le immagini più significative. 
convenzioni col Governo. Con la prima si obbligava a costruire e immergere fra l'Italia e le isole 12 cavi sottomarini per una lunghezza complessiva di circa $800 \mathrm{~km}$ e a curarne poi la manutenzione in perfetto stato per 20 anni. Con la seconda si obbligava a fare costruire, a proprie spese, un piroscafo attrezzato e capace di portare almeno 400 tonnellate di cavo (cioè circa $400 \mathrm{~km}$ ) e di consegnarlo alla R. Marina. Questa, a sua volta, doveva mettere il piroscafo a disposizione della Ditta Pirelli per i lavori relativi ai cavi, provvedendo equipaggio, ufficiali e dotazioni, mentre la Pirelli avrebbe dovuto provvedere il personale tecnico specializzato; e questo per 20 anni, dopo i quali sarebbe passata in proprietà alla R. Marina.

Intanto la Pirelli provvedeva alla costruzione dello stabilimento in riva al mare a S. Bartolomeo presso La Spezia. Era questo il primo stabilimento del genere che sorgesse sul continente europeo.

La nave, battezzata poi Città di Milano, fu ordinata ai Cantieri di Sunderland (Inghilterra) specializzati in questo genere di costruzioni e fu varata verso la fine del 1886. Ecco le sue caratteristiche principali: Lunghezza $\mathrm{m}$ 70, Larghezza interna massima m 9,75, Tonnellaggio lordo Tonn. 1220, Netto Tonn. 720, Motore a vapore da 1000 cavalli, Velocità oraria miglia 11. Le vasche, circolari, destinate a contenere immersi in acqua di mare e côlti in strati orizzontali (non su bobine), i cavi già preventivamente giuntati in fabbrica prima d'imbarcarli, erano tre, del diametro di circa 7 metri, capaci di contenere circa $400 \mathrm{~km}$ di cavo, tipo di fondo, il più sottile, del diametro esterno di circa $20 \mathrm{~mm}$ e del peso di circa $1 \mathrm{~kg}$ per metro. Un sistema di guide e di rulli conduceva il cavo agli argani della macchina di posa per svolgerlo, alle due puleggie di prua o a quella di poppa, passando per un dinamometro che ne misurava lo sforzo durante i lavori di posa e di rilevamento. La nave, per l'epoca in cui venne costruita, era una delle migliori ed era munita di tutta l'attrezzatura meccanica ed elettrica più perfezionata.

Mentre la Pirelli si stava preparando, un luttuoso avvenimento in Eritrea colpiva il nostro Paese; a Dogali il 27 gennaio $1887^{3}$ una nostra colonna veniva sopraffatta e distrutta malgrado l'eroica resistenza. Si mostrò così l'urgenza di stabilire una comunicazione telegrafica con la nostra Colonia primogenita allacciando Massaua ed Assab con l'isola di Perim (Stretto di Bab-el-Mandeb) toccata da cavi telegrafici inglesi che risalendo il Mar Rosso, attraverso l'Egitto, poi in Mediterraneo, passando per Malta, arrivavano anche in Sicilia.

Il Governo fece urgente appello alla Pirelli per la fornitura e la posa di circa $700 \mathrm{~km}$ di cavo. Dopo 2 mesi essa, non essendo ancora pronta, dovette ricorrere a una società

3 Nel dattiloscritto si leggeva 17 gennaio 1887. 
inglese, la quale fornì parte del cavo, la nave posa-cavi ed il personale specializzato inglese. Presero parte, imbarcati su questa nave, l'Ing. Pirelli e l'Ing. Emanuele Jona, giovane ingegnere elettricista italiano appena assunto e che era destinato a diventare Capo del servizio cavi sottomarini ed a legare a questo, per molti anni, il suo nome. I cavi del Mar Rosso vennero posati fra il 20 e il 29 marzo 1887, e quindi assai prima della data fissata dal contratto.

Nel giugno 1887 la Città di Milano era pronta ed iniziò la sua gloriosa opera con la riparazione di un vecchio cavo inglese posato per conto del governo Borbonico di Napoli nel 1860 fra Otranto e Valona. In seguito proseguì all'immersione dei cavi, previsti dalla Convenzione, fra l'Italia e le Isole, terminando, con varie campagne, susseguitesi senza interruzione, nel luglio 1888. A queste parteciparono, oltre all'ing. Pirelli, l'ing. Jona, l'ing. Ettore Pinelli, divenuto poscia Direttore dello Stabilimento di Spezia, e l'ing. Leopoldo Emanueli che fu in seguito Capo del Riparto Conduttori dello stabilimento di Milano.

Tutto il Paese aveva seguito con vivo interesse le notizie date alla stampa sui lavori di completamento della rete sottomarina e l'ing. Pirelli ebbe la soddisfazione di ricevere molti telegrammi inneggianti "alla Ditta Pirelli promotrice di civiltà".

Oramai la Pirelli poteva, con tutta tranquillità, cimentarsi in qualunque lavoro di questo genere, e nel giugno 1888 riusciva vincitrice, in concorrenza con le maggiori case inglesi, in una gara indetta dal Governo spagnolo per la posa di un cavo tra la Spagna e le Isole Baleari. Andato bene anche questo lavoro, nel 1890, vinse un'altra gara indetta dal Governo spagnolo per la posa di 7 linee che dovevano congiungere la Spagna col Marocco e con Tangeri per una lunghezza complessiva di $620 \mathrm{~km}$. La posa, particolarmente difficile, per la natura del fondo e le correnti dello Stretto di Gibilterra, riuscì felicemente, anche questa diretta dall'ing. Pirelli, dimostrando che gli italiani non erano secondi a nessuno in questo difficile ramo dell'industria elettrica.

\section{2. [Mar Rosso, isole greche, terremoto di Messina]4}

La Città di Milano proseguì poi la sua tranquilla opera di manutenzione dei cavi già posati e di posa di altri cavi con la Sardegna, la Sicilia e le Isole minori. I cavi del Mar Rosso, posati in un mare caldo e poco profondo, si guastavano facilmente, tanto da richiedere una o anche due campagne di riparazione all'anno.

\footnotetext{
$4 \mathrm{Nel}$ dattitloscritto il capitolo era privo di titolo. Lo abbiamo integrato considerando gli argomenti in esso trattati.
} 
Nell'estate 1900, trovandosi appunto in Mar Rosso per riparare il nostro MassauaAssab-Perim, diede occasione alla Compagnia inglese dei Cavi Telegrafici di affidarle la manutenzione dei suoi cavi sia in Mar Rosso che in Oceano Indiano. Rimanendo di stazione ad Aden nei periodi di sosta, in attesa di prestare la sua opera, questa le venne appunto richiesta per la riparazione del cavo Aden-Bombay, del Perim-Obok-Gibuti e di altri cavi minori.

Venuto l'autunno e con esso il periodo del Monsone - fortissimo vento dell'Oceano Indiano - che rende molto laboriosa e difficile la navigazione per parecchi mesi anche nella parte meridionale del Mar Rosso, la Città di Milano lasciò i mari tropicali per tornare in Mediterraneo, dove l'attendevano altre riparazioni su cavi della Compagnia inglese.

Prima di lasciare il Mar Rosso credo opportuno ricordare un episodio.

Durante una riparazione del cavo Massaua-Assab, sorpresi dal cattivo tempo, che impediva i nostri lavori, andammo a rifugiarci alla Baia d'Anfila, una insenatura ampia cosparsa di isolette madreporiche. Eravamo da tempo senza viveri freschi e si pensò di andare a terra a cercare di comperare qualche capretto od altro. Si decise di inviare due imbarcazioni: una, la barca a vapore, col personale militare; l'altra, una grossa barca a vela, con personale borghese. La barca a vapore partì seguendo una rotta dove i fondali lo permettevano, la nostra barca seguendone un'altra più breve ma con fondali minori. Navigando alla vela e quindi senza far rumore, rasentammo alcuni di questi isolotti resi verdeggianti da una bassa vegetazione e qui ammirammo a migliaia stormi d'uccelli trampolieri dai colori vari e vivaci, per nulla spaventati dal nostro silenzioso passaggio.

Arrivando in prossimità del villaggio indigeno, costituito da un centinaio di tucul fra palme da datteri, ci dovemmo fermare all'inizio del bassofondo, ove era già ancorata la barca a vapore. Alcuni nativi con le loro canoe, imbarcazioni primitive costruite da tronchi di palme scavati, vennero a prenderci e portarci a terra. Qui a mezzo di un indigeno, ex-ascaro che faceva da interprete, trovammo quanto cercavamo. Intanto, essendosi diffusa la notizia del nostro arrivo, tutta la popolazione era accorsa alla spiaggia; e poichè c'era un ammalato, nella convinzione che un tenente di vascello giunto con la barca a vapore fosse un akim cioè un dottore, insistettero perchè lo visitasse. L'ammalato, un giovanottone, si toccava la pancia, piuttosto gonfia; il male era lì. Il tenente, piuttosto imbarazzato, gliela palpa, poi gli fa capire di mostrargli la lingua, poi rivolgendosi a me, dice: "Caro Del Grande, qui stiamo passando un guaio; come si può capire, guardando la lingua di un nero, se è sporca o no? Ad ogni modo dobbiamo cavarcela" e poi volgendosi ad un marinaio: "Di', marinaio, vai a bordo della barca a vapore e portami l'oliatore”. Avutolo, fece aprire la bocca al disgraziato 
versandogli alquanto del contenuto ed esclamando: "Forse un po' d'olio di ricino qui dentro ci sarà!”.

Per compenso il Capo del villaggio ci regalò una cesta di foglie di palma intrecciate, contenente dell'ottima polpa di datteri, che fu assai gradita a bordo. Abbiamo poi saputo che, per renderla stagna, la cesta era intonacata all'interno con sterco di cammello!

Abbiamo anche saputo più tardi che il disgraziato era guarito.

Ed eccoci con la nostra nave, dopo il suo $20^{\circ}$ passaggio del canale di Suez, in Mediterraneo, dove ci accoglie un vento fresco da tramontana; passiamo così, in pochi giorni, da $48^{\circ} \mathrm{C}$ all'ombra, di Aden (un proverbio arabo dice: Aden un forno, Massaua un inferno!), ai pochi gradi sopra zero del mare Egeo in dicembre.

Arriviamo quindi, piuttosto intirizziti, di sera, nel porto di Syra, una piccola, ma importante isola greca, dove approdano i cavi con le molte isole dell'Arcipelago Greco. Vengono tosto a bordo due rappresentanti della Compagnia inglese dei cavi per accordi sui lavori da farsi cominciando dall'indomani. Scendono poi all'albergo per passarvi la notte, lasciandoci in consegna un astuccio di metallo contenente le carte nautiche con ivi segnati i tracciati di tutti i cavi costituenti la vasta loro rete in Mediterraneo.

Poichè il tracciato dei cavi sottomarini è tenuto segreto per ovvie ragioni militari, quella notte, chi scrive queste note non dormì, per trarne copia. Cosa che servì poi, come vedremo in seguito, a permetterci di tagliare a colpo sicuro, anche di notte e in alto mare, le comunicazioni nemiche durante le guerre che in seguito scoppiarono.

L'ultimo dicembre del secolo scorso venne passato riparando parecchi cavi in Arcipelago greco, alcuni dei quali raggiungevano le isole turche come Rodi, l'Asia Minore, Smirne e i Dardanelli.

Ai primi di gennaio 1901, rientrata nel mare nostro, procedette alla riparazione di un vecchio cavo posato dagli inglesi fra Orbetello e la Sardegna nel 1875: riparazione che riuscì alquanto laboriosa. In febbraio, sempre per conto della Compagnia inglese, si iniziò la riparazione del cavo Marsiglia-Barcellona guasto a circa metà percorso e precisamente in mezzo al Golfo di Lione, di famigerata nomina per le sue tempeste. Anche qui, malgrado molte traversìe dovute al mal tempo, sopportate in modo brillante dalla nostra bella nave, le cose finirono bene ed ai primi di marzo eravamo di ritorno a La Spezia, da dove eravamo partiti nel giugno dell'anno precedente.

Il 28 dicembre 1908 avvenne il terribile terremoto di Messina. Ne furono vittime pure i numerosi cavi telegrafici che attraversano lo Stretto di Messina, tutti interrotti perchè le loro estremità erano state strappate dagli approdi dalle formidabili onde di maremoto che flagellò quella notte le coste dello Stretto. Fu tosto organizzata d'urgenza 
una spedizione della Città di Milano, per procedere alle riparazioni, ed ai primi del gennaio 1909 la nave era a Messina. Abbiamo trovato la bella e ospitale città, che avevamo lasciato una quindicina di giorni prima per rientrare alla Spezia, completamente distrutta! Come uno scenario rimaneva in piedi solo la facciata della famosa Palazzata che circondava il porto, e che nascondeva le rovine della città.

Oltre ad accudire ai lavori di riparazione, ostacolati dal maltempo che si accaniva con pioggia, grandine ed anche neve contro la disgraziata popolazione superstite, nei frequenti attracchi sulla costa Calabra cercavamo di portare aiuti e soccorsi di viveri da Scilla a Catona.

La nave in porto a Messina si ormeggiava ad una boa posta al centro del porto stesso, dove la profondità è di 60 metri; siccome le scosse di terremoto continuarono per più settimane, quando queste avvenivano, specialmente di notte, generalmente precedute da un boato, malgrado il grosso cuscino d'acqua che avevamo sotto, il bastimento era fortemente scosso e tutto vibrava a bordo. In città intanto altri muri rimasti in piedi cadevano, rendendo pericolosissima la circolazione sulle macerie. Ricordo poi il lezzo dei cadaveri, tanto forte che, anche otto giorni dopo lasciata Messina, si sentiva ancora a bordo entrando in camerino.

Nei depositi di carbone del porto intanto covava un incendio. Vicina vi era immagazzinata una forte quantità di latte di benzina che occorreva allontanare e all'uopo si era ormeggiato vicino un grosso veliero. Avvenne un forte scoppio. "Incendio a bordo" venne tosto segnalato. Noi mandammo dei nostri, tra cui qualcuno col seghetto per tagliare eventualmente la catena dell'àncora e poi rimorchiare il veliero fuori del porto; ma il calore non permise di avvicinarsi. Una torpediniera lo cannoneggiò per affondarlo, ma lo scafo, essendo di legno, continuò a galleggiare. Noi avendo una caldaia in avarìa, avevamo i fuochi spenti e non potevamo lasciare l'ormeggio della boa. L’incendio continuò violento tutta la notte bruciando lo scafo del veliero man mano che per alleggerimento esso si alzava dal pelo dell'acqua, finchè verso le ore 10 del mattino la chiglia si sprofondò lasciando galleggiare numerose casse di legno contenenti le latte di benzina incendiata. Queste portate dalla corrente iniziarono il giro del porto, con grave minaccia specialmente per un bastimento in disarmo carico di profughi, donne, vecchi e bambini; e fu allora un accorrere dei nostri con tutti i mezzi disponibili, per portarli in salvo; manovra felicemente riuscita. Continuando il giro del porto e sfiorando depositi di nafta della R. Marina, la corrente portò fuori a disperdersi nello Stretto le ultime casse galleggianti. Finalmente ogni pericolo era scongiurato, ma se avesse preso fuoco la nafta, sarebbe stato un disastro! Tutto il porto si sarebbe convertito in un rogo. 
A riconoscimento delle benemerenze, la Città di Milano ebbe poi dal Ministero dell'Interno la Menzione Onorevole, e l'equipaggio l'autorizzazione di fregiarsi della medaglia commemorativa.

\section{Partecipazione della Città di Milano alla guerra italo-turca (1911-1912)}

Il 27 settembre 1911, allorchè il governo italiano diede l'ultimatum a quello turco, la R.N. Città di Milano era già partita dalla Spezia col programma di precedere la Squadra navale italiana nelle acque di Tripoli per tagliare il cavo Tripoli-Malta. Ma mentre navigava nello Stretto di Messina, si manifestò un'avarìa in macchina che la costrinse a poggiare nel porto di Augusta per un giorno. Intanto la squadra partiva e il 29 settembre, a guerra dichiarata, dopo un breve bombardamento di Tripoli, questa città alzava bandiera bianca. Quando noi giungemmo non era più necessario tagliare il cavo con Malta e fummo utilizzati per una missione alquanto inaspettata. L'entrata del porto di Tripoli era stata ostruita dai turchi autoaffondando il loro trasporto Derna che, sfuggito alla vigilanza dei nostri, vi era giunto qualche giorno prima carico di armi e munizioni. Il corpo d'occupazione doveva quindi essere sbarcato sulla spiaggia di Gargaresch, ma mancavano i mezzi di sbarco. Ebbimo allora l'incarico di recarci nelle acque dell'isola di Lampedusa ed ivi requisire la numerosa flottiglia di barche, dette coralline, colà intente alla pesca di spugne e coralli; ne trovammo una cinquantina che, prese a rimorchio, portammo a Tripoli ove servirono egregiamente.

Dopo l'occupazione di Bengasi, ai primi di Novembre, venne proclamata l'annessione della Libia. Si pensò allora di collegare telegraficamente Tripoli e Bengasi con l'Italia, e la Ditta Pirelli ebbe l'incarico di provvedere alla posa dei cavi SiracusaTripoli e Siracusa-Bengasi, rispettivamente della lunghezza di km 550 e 750 circa. La lunghezza del cavo di Bengasi era troppo forte per la capacità delle vasche della Città di Milano e siccome vi era urgenza e non sarebbe stata consigliabile la posa in due o tre viaggi consecutivi, la Ditta Pirelli noleggiò in Inghilterra la nave Cambria sulla quale presi imbarco insieme coll'ing. Jona, per posare tutta la parte centrale del cavo Siracusa-Bengasi, con la Città di Milano avendo già posate le parti riguardanti i due approdi. 
Nell'aprile5 1912, dichiarato il blocco delle isole turche dell'Egeo, mentre la nostra nave stava caricando a La Spezia il cavo occorrente per la linea Siracusa-Tripoli, dovette sospendere tale lavoro per recarsi in Egeo a tagliare i cavi turchi. L'appuntamento era di aggregarci alla nostra squadra navale che si recava ai Dardanelli per un'azione contro i forti e contro la squadra turca ivi rifugiata a Kumkalé. Giunti in Egeo, al luogo fissato, prendemmo posto, in formazione, sulla destra della nave ammiraglia Vittorio Emanuele dirigendoci con un ampio giro in acque greche e, con mare alquanto agitato dal vento greco di tramontana, per i Dardanelli; giunta la notte, in prossimità dei Dardanelli la squadra ci lasciò, scortati da un cacciatorpediniere, con l'incarico di tagliare il cavo Imbro-Dardanelli. Notte buia e tempestosa, tanto che disperavamo di individuare il punto ove pescare il cavo da tagliare. Naturalmente a bordo c'era oscuramento completo. Verso mezzanotte ecco accendersi all'improvviso due proiettori, che tosto individuammo appartenere ai due forti dell'imboccatura dei Dardanelli. Potemmo allora fare il punto e prendere posizione per il grappinaggio del cavo, che allora pescammo da una profondità di circa 500 metri asportandone 500 metri. Mentre si stava salpando, uno dei proiettori dirigeva su di noi il fascio luminoso; furono pochi istanti di grande ansia per noi! Se i turchi avessero capito che cosa stavamo facendo, ci avrebbero certamente presi a cannonate. Invece niente.

Intanto una squadriglia di torpediniere nostre avrebbe dovuto forzare l'ingresso dei Dardanelli, certa di non essere scoperta dal nemico, poichè una spia tedesca, imbarcata sulla capo-squadriglia (ed era un ufficiale superiore tedesco che diceva aver preso parte ai lavori di fortificazione), aveva assicurato che i turchi non avevano in dotazione proiettori elettrici. Naturalmente la loro missione fallì, perchè alla vista dei proiettori le torpediniere invertirono la rotta dirigendo per la baia Mudros nell'isola di Lemno, dove era la nostra squadra, la quale, informata dal nostro caccia di scorta del felice esito del nostro lavoro, il mattino seguente si portò davanti ai forti iniziandone il bombardamento.

Per essere preciso aggiungerò che la missione delle torpediniere, fallita quella volta, fu poi ripetuta con esito brillante più tardi, al comando dell'Ammiraglio Millo.

Il giorno dopo noi pescammo il cavo Tenedo-Lemno. Appena avutolo a bordo e tagliatolo, per assicurarci che fosse un cavo attivo e non uno spezzone abbandonato durante una riparazione, lo attaccammo all'apparato telegrafico Morse, col quale cominciai a fare degli interrogativi, ai quali il telegrafista turco di Tenedo rispose subito con dei "Chi là? Eastern là?".

5 Nel dattiloscritto si leggeva Nel maggio 1912. Fu invece nell'aprile del 1912 che la R.N. Città di Milano fu impegnata nel taglio dei cavi turchi. 
Il Comandante, che era il Capitano di Corvetta Guido Milanesi - il noto scrittore di romanzi e novelle a soggetto marinaresco -, mi suggerì di chiedergli in inglese se aveva notizie (Have you news?) al che avemmo risposta in italiano: "Niente, caro signore". Risata generale di tutti i presenti e il Comandante rivolto a me: "Si vede che l'ha capito all'accento". "Se mai” risposi “avrebbe dovuto esprimersi in milanese!”.

Il giorno dopo, pescato il cavo Tenedo-Metelino, si ripete la scena; agli interrogativi nostri il turco chiede il "Chi là?" al che io rispondo: "Siamo ancora gli amici di ieri”. E lui: "Sempre amici, caro signore". Anche qui, tagliati via circa 500 metri, buttammo le cime a mare.

L'indomani fu la volta dei tre cavi tra l'isola di Chio e la stazione di Casmé sulla costa dell'Asia Minore vicino a Smirne.

Intanto, avendo il Governo Austriaco messo il veto alle nostre operazioni di guerra al disopra del $38^{\circ}$ parallelo, ebbimo l'ordine di rientrare a La Spezia per completare il carico del Siracusa-Tripoli. Operazione dovuta ancora interrompere, per recarci a tagliare il cavo Rodi-Sitia (Creta), all'altezza dell'isola di Scarpanto.

Durante la navigazione il Comandante (Milanesi) credette opportuno camuffare la nave in modo da renderla irriconoscibile, sporcando il fuori-bordo, mettendo un palo sporgente alla prua in modo da simulare un bompresso, verniciando la ciminiera con una striscia bianca con una palla rossa ecc. Passate le isole greche di Cerigo e Cerigotto le vedette scorgono sulla sinistra un grosso vapore, e allora il Comandante, nel timore che fosse una nave nemica, ordina di aumentare la velocità e di deviare dalla rotta per buttarci nelle acque neutrali di Capo Spada (Candia). Ma quel vapore, essendo molto più veloce di noi, c'insegue, finchè avvicinandoci riusciamo a individuarlo per il nostro incrociatore ausiliario Duca degli Abruzzi. Questo presto ci raggiunge, per chiedere se venendo dall'Italia avevamo giornali freschi, loro essendo da più di un mese là in crocera e quindi privi di notizie. Ci avevano quindi riconosciuti subito, malgrado il nostro camuffamento!

L'indomani mattina, all'albeggiare, arriviamo davanti a Scarpanto, dove ci attendeva l'incrociatore Coatit per scortarci durante i lavori. Un ufficiale venuto a bordo ci disse che l'avevamo scampata bella. Il Comandante del Coatit quando ci avvistò non ci aveva riconosciuto ed aveva messo la gente al posto di combattimento. Per fortuna, avendo noi deviato dalla rotta, avevamo poi mostrato il fianco e con esso la bandiera che sventolava a poppa! Se no, ci avrebbe preso a cannonate.

Eseguito il taglio, altro ritorno in Italia, con tappa ad Augusta. Intanto era avvenuto lo sbarco dei nostri a Rodi al comando del Generale Ameglio, il quale dopo la battaglia di Psitos (16 maggio), per corrispondere meglio con Roma, chiese il ripristino del cavo 
con Candia, cavo che, allacciato con Zante e poi con Otranto, arrivava in Italia. Dovemmo quindi ritornare in quelle acque. La riparazione, trattandosi di un vecchio cavo dall'armatura corrosa che continuava a strapparsi durante il salpamento, fu piuttosto laboriosa, tanto che per colmare la lacuna di 500 metri fatta da noi, impiegammo poi 25 chilometri di cavo nuovo.

\section{Partecipazione della Città di Milano alla Grande Guerra}

\section{(1915-1918)}

Il 22 maggio 1915 la Città di Milano stava riparando il cavo Lipari-Filicudi. Pescatolo e tiratolo a bordo, la cima verso Lipari venne attaccata alla linea del gabinetto elettrico di bordo per essere provata. Era buona; il guasto era quindi verso Filicudi. Attaccata all'apparecchio Morse per trasmettere istruzioni al telegrafista di Lipari, questo avverte subito di avere un telegramma cifrato urgente di Stato per il Comando della nave. Avverto di ciò il Comandante, che sta sulla plancia, dicendogli se devo riceverlo io stesso o se voleva mandare il suo R.T. Mi rispose che lo ricevessi pure io. Gli passai poi il gruppo di cifre trasmessomi, che egli subito a mezzo del codice segreto in suo possesso mise in chiaro. Era l'ordine, da parte del Ministro della Marina, di sospendere i lavori e di recarci subito a Messina ove avremmo avuto istruzioni. E ciò egli comunicò al nostro Capo spedizione Ing. Jona, il quale ordinò tosto di mettere in mare le due cime del cavo affidandole a due boe.

Arrivammo in porto a Messina la sera, e tutta la notte imbarcammo carbone, acqua, viveri. L'indomani, giorno della mobilitazione generale, partenza per destinazione ignota. Girando Capo Spartivento, ci voleva poco a capire che eravamo diretti in Adriatico. Mentre eravamo in navigazione, all'altezza di Capo Colonna, ci raggiunse un cacciatorpediniere, il quale, con agile manovra, ci affiancò senza che noi fermassimo, e un marinaio salì al nostro bordo con una grande busta gialla, sigillata; era l'ordine di recarci in Adriatico per tagliare il cavo austriaco Trieste-Corfù. Era questo l'unico cavo rimasto in attività dei nostri nemici d'allora e che dava loro la possibilità di comunicare col mondo. I cavi tedeschi a nord erano stati tutti quanti tagliati dagli'inglesi poche ore dopo la dichiarazione di guerra con la Germania; e quelli che, passando per la Manica, univano la Germania all'America, furono deviati e fatti approdare in Inghilterra. L'Italia intanto dichiarava la guerra all'Austria (24 maggio).

Proseguendo, con circospezione e con rotta di sicurezza, la mattina del 25 maggio, al largo di Capo di Santa Maria di Leuca, ci raggiunse una squadriglia di cacciatorpediniere, Indomito, Intrepido, Impavido e Irrequieto (in Marina la 
chiamavano senz'altro la squadriglia degl'impestati) che ci doveva scortare. Giunti nel porto fissato, in canale di Otranto, verso la mezzanotte del 25, iniziammo tosto il grappinaggio del cavo a bordo. Assicuratici che era un cavo in funzione, ne asportammo circa 500 metri, quindi buttammo le cime in mare. Un elettricista di cattivo carattere avrebbe potuto applicare alle due teste del cavo una resistenza, in modo da falsare eventuali ricerche del guasto da parte del nemico; ma tanto sarebbe stato inutile, poichè sapevamo che l'Austria non aveva mezzi per poter ristabilire la comunicazione.

Dirigemmo quindi per Taranto. Girando Capo Santa Maria di Leuca, verso le due del pomeriggio, all'altezza di Gallipoli, mentre noi, che avevamo passato gran parte della notte in bianco, eravamo sdraiati a riposare, fummo svegliati da colpi di cannone (a bordo avevamo 4 cacafuoco, 2 da 75 e 2 da 57); saliti in coperta, ci venne detto come fosse stato avvistato un periscopio sulla sinistra e visto poi un siluro passare a tre metri dalla poppa della nostra nave! Intanto i caccia di scorta andavano zigzagando e sparando in mare, buttando anche qualche bomba. La nostra velocità era stata fino allora inferiore alle 10 miglia: causa il carbone cattivo, dicevano in macchina; ebbene, la velocità nostra da questo momento andò aumentando fino a raggiungere le 12 miglia: il buon volere dei fuochisti aveva migliorato il combustibile!

Forse uno dei caccia aveva investito il sommergibile, avendo esso sentito un urto. Infatti, andato poi il caccia in bacino a Taranto, fu riscontrata una forte ammaccatura nella chiglia: forse abbiamo avuto così l'onore d'essere noi il bersaglio del primo siluro austriaco.

Dopo qualche giorno di permanenza a Taranto, dove trovammo ormeggiate in Mar Piccolo navi da guerra francesi ed inglesi, fummo lasciati liberi di ultimare la interrotta riparazione del cavo Lipari-Alicudi, ritornando in seguito a La Spezia.

Altre riparazioni si fecero in seguito, sempre scortati da caccia o da Mas. Notevole quella dei cavi a nord dello Stretto di Messina fatta nel dicembre 1916. Erano questi di tipo speciale a tre conduttori, che la Compagnia inglese manteneva gratis, al Governo italiano, tra Bagnara Calabra e la Punta Faro di Messina, in compenso della concessione di approdo in Italia dei cavi della detta Compagnia a Modica in Sicilia, di quelli con Malta e di Otranto con le isole di Corfù e Zante.

A Messina prendemmo a bordo un elettricista inglese che era stato inviato da Malta da detta Compagnia per assistere ai lavori. Era un tipo di carattere gioviale e abbastanza simpatico. Alla mensa ufficiali noi avevamo dell'ottimo vino Chianti, che egli rifiutò perchè, diceva, gli metteva bruciori allo stomaco; preferiva il cognac, del quale consumava a tavola una bottiglia al giorno. In camerino poi ne teneva dell'altro, che sorbiva durante il giorno e la notte. Una volta mi fece capire di avere smarrito 
qualche cosa nel suo camerino; vi andai col cameriere e sotto la rete metallica della cuccetta trovammo una corona del Rosario! Ci spiegò che egli era uno scozzese di religione cattolica e che aveva sposato una maltese. I maltesi sono religiosissimi. Di modo che, quando durante i lavori frequentemente si ecclissava, noi si diceva: “L’inglese è andato a recitare qualche Avemaria!". Avendo sposato una maltese doveva capire l'italiano, ma con noi più del suo inglese non parlava. Un giorno stavamo passando, in coperta della nave, degli spezzoni di cavo salpato, per giudicare se erano ancora utilizzabili. Egli assisteva alla verifica, approvando i miei "good" cioè "buono", finchè, arrivati a un pezzo dall'armatura molto corrosa, lo giudicai "no good", "non buono", ed egli di rimando "very good", "ottimo", allora io rivolgendomi all'operaio capo-giuntista, che era milanese e che mi guardava stupito: "Mariani, content lu, content tutti!”. E mister Miall subito “Jes, mister Del Grande, contento lui, contenti tutti!". Quell'animale d'inglese aveva quindi capito il nostro milanese!

Ultimata la riparazione dei cavi dello Stretto ebbimo l'ordine di recarci in Adriatico per posare alcune linee strategiche davanti a Valona. Essendo sopraggiunte le feste Natalizie, a Messina al pranzo di Natale non ci mancò il tradizionale panettone, che la Ditta Pirelli, come d'uso, quando eravamo fuori, magari anche in Mar Rosso, era usa inviare per tutto l'equipaggio borghese e militare. E alla mensa ufficiali non mancò nemmeno il risotto, veramente alla milanese, al quale pensai io, sostituendo il cuoco che era napoletano.

La sera stessa dovevamo partire per Valona e l'equipaggio stava facendo i preparativi, mentre noi eravamo a pranzo. Verso la fine di questo, quando si sarebbe dovuto bere lo spumante, ecco scendere in quadrato ufficiali il capo cannoniere, il quale, dopo avere chiesto il permesso al Comandante, aprì la botola della Santa Barbara, che stava sotto il nostro salone, e aiutato da alcuni marinai estrasse alquanti proiettili per i nostri cannoni, disponendoli verticali lungo le pareti come se fossero stati bottiglioni! Una fine del pranzo di Natale veramente allegra, non vi pare?

Partimmo quindi da Messina la sera stessa, da soli; la scorta ci avrebbe raggiunto più tardi verso Capo S. Maria di Leuca, cioè prima di entrare in Adriatico. Fuori dello Stretto, prendemmo il largo seguendo una prescritta rotta, detta di sicurezza perchè vigilata dalla nostra Marina. Il mare era alquanto agitato da un forte vento di tramontana.

In navigazione, durante la notte, ci si sdraiava vestiti in cuccetta e col salvagente sempre pronto a portata di mano, pronti a qualsiasi brutta eventualità.

L’indomani mattina, verso le ore 7,30, nel dormiveglia, sento fermare le macchine, poi metterle "indietro a tutta forza" per arrestare la nave. Corro subito in coperta e 
chiedo in plancia che cosa c'è. "Niente", mi risponde l'ufficiale di guardia, "la vedetta di coffa ha avvistato di prua qualche cosa di sospetto che galleggia. Ora aspettiamo che si faccia un po' di chiaro e vedremo". Dopo un'oretta di attesa, fattosi più chiaro, col binoccolo scorgiamo a circa 500 metri dalla prua un grappolo di 4 mine! $\mathrm{Ci}$ avvicinammo manovrando adagio e coi moschetti alcuni marinai tirarono alle camere d'aria delle mine per perforarle; l'acqua entrando le appesantì e in pochi momenti colarono a picco. Se le avessimo incontrate solo un'ora prima, quand'era ancora buio, forse non sarei più qui a raccontarvela.

Giunti il giorno dopo davanti alla baia di Valona, approfittando del mare tranquillo, procedemmo alla posa delle linee strategiche, fuori dello sbarramento di mine, fra l’isolotto di Saseno, Capo Linguetta e Capo Treporti.

Verso sera, a operazioni finite, stavamo girando a levante di Saseno per entrare a Valona, quando il bastimento si ferma malgrado che le eliche girassero sempre. Eravamo incagliati su di un bassofondo, per nostra fortuna solo fangoso, non segnato nelle carte nautiche! Cercammo di disincagliarci mettendo inutilmente le macchine indietro a tutta forza. Che fare? Un rimorchiatore che ci aiutasse non era facile averlo. Pensammo allora di ricorrere al nostro potente argano per salpare cavi ed agli [altri] ${ }^{6}$ nostri mezzi a disposizione.

Caricato su di una barca un ancorotto a fungo, assicurato ad un cavo di manillaacciaio, lo buttammo in mare a circa 500 metri dalla poppa nostra, poi, messo in moto l'argano, cominciammo a virare, mentre l'elica faceva marcia indietro. Presto la nave prese a muoversi indietreggiando ed in poco tempo fummo liberi.

Intanto s'era fatto buio. Il Comandante, un uomo calmo, ma in gamba, che mi piace qui ricordare e che era il Capitano di Corvetta Spano Fermo, radunò noi dello Stato Maggiore e ci disse: "Cari amici, so che è rischioso passare col buio lo sbarramento di mine, indovinandone l’ingresso, che è solo di 200 metri di larghezza. D’altra parte rimanere qui fuori è assai pericoloso, perchè di notte vengono sommergibili austriaci a deporre le loro uova (cioè le mine). Se ci incocciano, le buschiamo. Io tento di entrare; non vogliatemi male se succedesse qualche disgrazia!”. E siamo felicemente entrati grazie al suo buon occhio nell'apprezzare la distanza dall'altura di Capo Linguetta.

Poche sere dopo, la corazzata Regina Margherita, nell'uscire, investì con la poppa le mine, saltò per aria ed andò perduta con quasi tutto l'equipaggio.

Rientrammo poi a La Spezia in attesa di altri ordini.

\footnotetext{
${ }^{6}$ Nel dattiloscritto si leggeva ed agli nostri mezzi da qui la nostra integrazione di altri.
} 
A differenza di quanto succede durante la guerra attuale, durante la quale i nostri colleghi Pirelliani imbarcati sulle navi posa-cavi sono stati militarizzati, noi non lo eravamo, e portavamo il distintivo di appartenenti a stabilimenti ausiliari, come quelli che rimanevano a Milano; gli esonerati, come nel caso mio, portavano il bracciale come un imboscato qualunque; ed era buffo, per esempio, vederci circolare, magari per Valona, con tale contrassegno.

Visto che il Governo non pensava affatto alla nostra situazione speciale, il Senatore Pirelli dispose che almeno da parte della Ditta ci fosse data una soddisfazione morale facendo coniare 5 medaglie d'oro ricordo per noi impiegati e 25 d'argento per gli operai imbarcati, medaglie che poi egli ci distribuì.

Purtroppo le vicende future fecero sparire tragicamente gran parte di noi della vecchia guardia, ed ora l'unica medaglia d'oro superstite è chi scrive, non privo di mestizia, questi ricordi.

Nel giugno del 1917 uno scoppio di munizioni avvenuto in prossimità del nostro stabilimento di San Bartolomeo (La Spezia) lo demolì quasi totalmente (rimase sana solo la ciminiera, che cadde poi, qualche anno dopo, causa di un terremoto). Le vittime furono più di 400, fra cui parecchi nostri animosi compagni di lavoro che erano accorsi per portare aiuto. Ciò provocò una sosta forzata alla nostra attività, cioè sino a che, ricostruitolo, si potè riprendere la fabbricazione dei cavi sottomarini.

Ed eccoci arrivati all'ultima campagna di guerra per la riparazione del vecchio cavo Orbetello-Sardegna, guasto nelle vicinanze delle Bocche di S. Bonifacio.

Partiti da La Spezia agli ultimi dell'ottobre 1918, la notte del 4 novembre stavamo ultimando la riparazione con la confezione del così detto giunto finale, che costituisce il coronamento del nostro faticoso lavoro. Verso le 2 del mattino viene a prua il marinaio marconista in cerca del Comandante che era lì con noi, e gli dice: "Signor Comandante, succede qualche cosa di straordinario che non capisco. Ho intercettato a stento un R.T. del Comando della flotta Jugoslava di Pola, diretto alla nostra stazione di Ancona, chiedendo se è stato inoltrato al Presidente Wilson un precedente suo telegramma”. Al che il Comandante, rivolgendosi stupito anche a noi parimenti stupiti: "Il Comando della flotta Jugoslava? Ma che roba è? Torna bene in ascolto e riferisci”. Poco dopo eccolo di nuovo: "Sig. Comandante, ora la flotta jugoslava di Pola ha chiamato il Comando della flotta francese ancorata a Corfù, ma non ho capito bene cosa ha detto. Ho inteso poi la risposta del comando francese, le soleil de la liberté vient de paraître!, cioè sta sorgendo il sole della libertà, poi più niente perchè disturbato da un S.O.S. lanciato da un piroscafo italiano nelle vicinanze di Capo Corso". 
Finito e buttato a mare il giunto finale, ci mettemmo in rotta per Porto Santo Stefano (Orbetello), ove giungemmo nel tardo pomeriggio. E qui eccoci un'altra sorpresa. Trovammo le vie del paese tutte imbandierate, mentre una processione religiosa sfilava con l'accompagnamento della banda musicale! Mentre ci stavamo ancorando, ecco che molti dei componenti la processione si staccano correndo verso la banchina, vociando e gesticolando verso la nostra nave. Il Comandante, col megafono, riesce ad ottenere che uno solo di essi parli e spieghi cosa vogliono da noi. "Come, siete una nave da guerra, e venite in porto senza il gran pavese a riva? Non sapete che l'Austria ha chiesto l'armistizio?”. Il Comandante rispose che avrebbe provveduto al più presto a fare preparare il gran pavese, che è formato da un centinaio di bandierine per segnalazioni giuntate una di seguito all'altra.

Potete immaginare con quale entusiasmo il nostro equipaggio si mise a prepararlo e, appena pronto, a issarlo sugli alberi della nave, mentre a terra la banda suonava la Marcia Reale e la popolazione applaudiva!

\section{Il naufragio della Città di Milano (16 giugno 1919)}

Nel giugno 1919 la Città di Milano dovette recarsi nelle acque delle Isole Eolie (Lipari) per riparare i cavi Vulcano-Lipari e Filicudi-Alicudi. Dopo riparato quello di Vulcano, essendosi messo cattivo tempo, andò ad ancorare nel porto di Milazzo.

Il mattino del 16 giugno 1919, il tempo essendo tornato buono, con mare calmo, lasciammo Milazzo dirigendoci verso l'approdo di Salina (Lipari) dove volevamo constatare le condizioni del cavo. L’ing. Jona m’incaricò di andare a vedere. Giunta a Salina verso le ore 11, la nave si fermò ed io scesi a terra con un battello; constatato che il cavo all'approdo era in buone condizioni, ritornai a bordo e la nave proseguì per l'altro approdo di Filicudi. È questa una isoletta di pochi chilometri quadrati, con circa 500 abitanti, di natura vulcanica, con la costa scoscesa e quindi senza fondali che permettano l'ancoraggio di vapori; intorno vi sono parecchi scogli a pochi metri sott'acqua, ed altri sporgenti, fra cui La Canna alto 75 metri, che visto un po' da lontano sembra una guglia del nostro Duomo.

Arrivati a Filicudi verso le ore 13, scesi anche qui a visitare approdo e casotto, e trovai tutto bene. Ritornai a bordo e riferii all'ing. Jona, che mi attendeva al barcherizzo; egli e il Comandante salirono in plancia (ponte di comando) e la nave, rimessa in moto, invece che girare a nord per recarsi all'altro approdo verso Alicudi, quello da riparare, perchè rotto per corrosione fra gli scogli, si diresse per passare a sud girando Capo Graziano: 


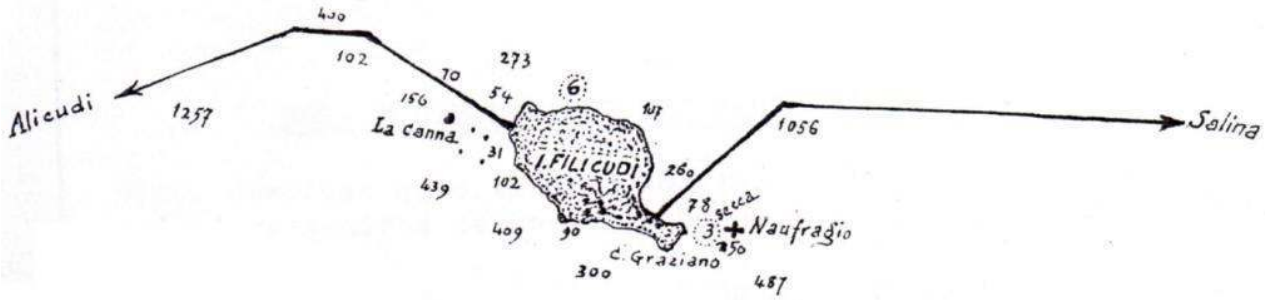

Io entrai in Gabinetto elettrico, posto sotto la plancia, e mi accingevo a provare lo spezzone di cavo robustissimo (a tripla armatura) destinato all'altro approdo, quando sentii due o tre scossoni, che fecero vibrare la nave; poi l'ordine in macchina di fermare. Allarmato mi girai. Sul tavolo stava stesa la carta nautica con segnata l'isola, le secche, gli scogli e le profondità. Intuii subito la situazione; avevamo urtato di striscio, lungo la secca di scoglio, circa 1000 metri distante da Capo Graziano, a 3 metri sotto l'acqua, ed eravamo fermi sopra un fondale di circa 250 metri. Decisi di correre a poppa, scendere in camerino e mettermi il salvagente di kapok. Andando verso poppa sentii il tenente che ordinava alla gente di mettersi in riga, e pensai: "Si quest chi el voer fag vosà, Viva l'Italia”. A poppa incontrai, spaventato, l'ispettore generale dei telegrafi, ing. Brunelli, al quale dissi: "Comendatur, sem a moi!" e lui "El dis davvera?”, "Si sul seri, alla svelta el salvagent, e tajem la corda!". Scendemmo tutti e due a prendere il salvagente in camerino. Tornato in coperta, vidi a poppa gli ing. Jona e Vitale [sic] perplessi, e, guardando lungo il bordo a sinistra notai che i nostri operai, trasgredendo gli ordini, calavano in mare la barca a vela, già quasi piena di gente; aiutai il Commendatore a scavalcare il parapetto e a filarsi giù lungo le corde del paranco e lo seguii. Intanto il bastimento aveva incominciato a inclinarsi a prua, l'elica era già fuori acqua; visto che non potevamo armare i remi per spostare dal fianco della nave, urlai “A mare!” e mi buttai, nuotando disperatamente per allontanarmi ed evitare di esser travolto dal gorgo. Non so dire quanto tempo sono rimasto in acqua: mi sentii poi chiamare per nome; mi girai e vidi un nostro battello con sopra, fra gli altri, il collega Capitano De 
Ferrari e il giuntista Lagomarsini, che avendo visto il mio berretto bianco mi avevano raggiunto. E mi tirarono a bordo. Il bastimento era scomparso, dopo lo scoppio delle caldaie che io, nell'orgasmo, nemmeno intesi. Vidi rottami cui erano attaccati dei naufraghi. Dirigemmo verso di loro e ne lasciammo aggrappare al nostro bordo il maggior numero possibile; per fortuna il mare era calmo come l'olio. Vidi la barca a vela capovolta, e sulla sua chiglia accoccolati due nostri operai che urlavano; dicemmo loro che andavamo a terra a Capo Graziano e che poi avremmo rimandato il battello per rimorchiarli. Intanto accorrevano dall'isola alcune barche di pescatori. Il battello ritornato al largo scorse l'ing. Pinelli, direttore dello stabilimento di La Spezia; era boccheggiante. Lo caricò e lo portò a terra; qui tentammo subito la respirazione artificiale, ma inutilmente. Egli, abile nuotatore, si era buttato a mare senza salvagente, ma, siccome avevamo appena fatto colazione, deve aver avuto un arresto nella digestione.

Rimorchiata a terra la barca a vela rovesciata, e raddrizzatala, ecco saltar fuori un uomo che si mette a scappare come un ossesso, e, sotto i bancali, il cadavere del Comm. Brunelli con un braccio rotto. Dal nostro più vecchio marinaio, Saioni, che era uno degli accoccolati sulla chiglia, ho poi saputo che quando il bastimento andò giù, le gru sporgendo avevano preso il bordo della barca rovesciandola. Egli e l'altro si erano poi sfilati nuotando e salendo sulla chiglia; al nostro passaggio ci chiamarono, perchè sotto sentivano urlare “Oh, Betta Matri7 (Beata Vergine), che morte mi tocca a fare!”. Era quel marinaio, un fuochista siciliano; che poi abbiamo visto scappare. Egli aveva potuto sopravvivere respirando l'aria rimasta sotto la chiglia della barca rovesciata.

Mentre eravamo al sole sugli scogli di Capo Graziano per asciugare i vestiti, barche e battelli portarono a terra i superstiti, tra cui il Comandante capitano di corvetta Cornigliani, l'ufficiale di rotta, il dottore e il direttore di macchina.

Ed ecco il triste bilancio: imbarcati uomini 101, superstiti 75, vittime 26, delle quali 24 scomparsi, 2 sole salme ricuperate, quelle dell'ing. Pinelli e del Comm. Brunelli. Del personale della Ditta Pirelli, morti: gl'ingegneri Jona Emanuele, Pinelli Ettore e Vitali Ettore, gli operai Galantini Libero, Porrini Giacomo e Tonelli Umberto. Salvi: il Geom. Del Grande Ernesto, il Capitano marittimo De Ferrari Stefano, il capo squadra giuntista Lagomarsini Primiero e 22 operai.

Urgeva dare notizia del disastro e chiedere soccorso. D’accordo col Comandante, mi recai io stesso all'Ufficio telegrafico lontano circa mezz'ora. Non trovai il telegrafista, che era ad attenderci all'altro approdo; v'era sua moglie, la quale mi rifocillò e mi diede anche una camicia di suo marito, la mia essendo, come il vestito, tutta imbrattata di

7 In siciliano è in realtà Bedda Matri. 
untume. Fattomi dare dall'Ufficio di Lipari la linea diretta con la Direzione dei Telegrafi di Messina, telegrafai io stesso al Comando Militare Marittimo di Messina, alla Ditta Pirelli a Milano e a La Spezia ed al Ministero delle Comunicazioni.

I soccorsi giunsero la sera tardi. Una torpediniera prese a bordo le due salme e parte dei superstiti. Verso mezzanotte arrivò da Milazzo un vapore, l'Adelaide, sul quale prendemmo imbarco; ci portò a Milazzo, da cui col primo treno andammo a Messina, dove fummo rifocillati ed anche vestiti. Molti dei naufraghi erano solo in mutande! Trattenuti qualche giorno per l'inevitabile inchiesta, fatta da un Ammiraglio, fummo finalmente liberi di tornare a casa nostra a rivedere le nostre famiglie.

Una quindicina di giorni dopo, io ero a Malta, per imbarcare su una piccola nave posa-cavi, il Levant Second della compagnia inglese, in rappresentanza della Ditta Pirelli e del Ministero delle Comunicazioni, per continuare i lavori interrotti col naufragio della nostra vecchia e cara Città di Milano.

\section{Posa e riparazione dei cavi sottomarini}

Crediamo opportuno, dopo aver parlato tanto dei cavi sottomarini, dare qualche cenno, alla buona, di questo argomento ignorato dai più.

Incominciamo col dire subito, per togliere una credenza errata abbastanza comune, che il cavo va ad adagiarsi, per il proprio peso, in fondo al mare, sia esso profondo 50100 metri, sia di 8.000 e magari 9.000 metri. In Mediterraneo la massima profondità, trovata posando il cavo Siracusa-Bengasi, è di 4.200 metri; in Atlantico 6.00o metri e nel Pacifico 9.00o metri. È ovvio che non si possono utilizzare i palombari anche lavorando in piccole profondità. Gli eventuali guasti vengono riparati pescando il cavo in mezzo al mare, nel punto del guasto; non si potrebbe prenderli alla spiaggia e salparli sino al guasto, l'operazione difficilmente riuscirebbe e guasterebbe anche il cavo.

Ed ora rechiamoci nel Golfo della Spezia a San Bartolomeo. Dolcemente cullata da leggera ondulazione, vediamo una nave di forme singolari; ha la prua molto slanciata, quasi come un becco d'anitra, che porta fuori tre massicce puleggie. Altra grossa puleggia vediamo sporgere dalla poppa; sul ponte, macchine ed ordigni svariati. È la R.N. Città di Milano, che si appresta ad una spedizione per lavori in cavi sottomarini.

Saliamo a bordo. La nave sta imbarcando diecine e centinaia di chilometri di cavo, di tipi diversi, che sono côlti, in strati orizzontali, in tre grandi vasche circolari. Vediamo imbarcare anche delle boe, di svariate forme e dimensioni, che servono a fissare un punto sul mare, ancorandole al fondo mediante cordami misti di acciaio e manilla, o ad assicurarvi la cima di un cavo telegrafico durante i lavori; vediamo una 
quantità di grappini (specie di ancorotti) di svariate dimensioni, che, mandati in fondo al mare, attaccati ad una corda di acciaio-manilla, servono a ricercare un cavo posato ed a sollevarlo alla superficie. Al centro della nave troviamo la macchina di posa composta di due tamburi indipendenti, azionati ciascuno da una macchina a vapore e muniti di freni potenti; essa serve alla posa ed al salpamento dei cavi. Allineati coi tamburi vediamo due dinamometri, e a poppa un altro, che misurano la tensione meccanica a cui è sottoposto un cavo durante i lavori. Al centro della nave troviamo pure il Gabinetto elettrico, ricco di svariati strumenti, dove si fanno le esperienze sui cavi per accertarne le buone condizioni o ricercare i guasti. A poppa troviamo pure una macchina per scandagliare la profondità del mare.

La nave dunque sta imbarcando del cavo e vediamo che essa ha steso, da uno dei suoi due alberi all'officina, una corda metallica che porta molte puleggine, entro le quali è infilato il cavo. Questo viene tirato a bordo con una macchinetta e inviato nelle vasche, dove viene disposto, come abbiamo detto, a strati orizzontali, guidato da un uomo che cammina per accompagnarlo. Operazione quindi abbastanza lunga, che può richiedere settimane di lavoro ininterrotto.

Vediamo un po' come è fatto un cavo telegrafico sottomarino. Anzitutto, il filo metallico che conduce la corrente nel cavo, è costituito da una cordicella di fili di rame; per isolare questa cordicella dal contatto dell'acqua, essa è circondata da uno o più strati di guttaperca e così costituisce l'anima del cavo. Occorre proteggere quest'anima da facili guasti, siano essi provocati dall'uomo (per esempio da àncore di navi, da draghe di pescatori) o da grossi animali marini, od anche semplicemente dal moto ondoso del mare e dalle correnti marine, che rimovendo continuamente l'anima e fregandola contro gli scogli, le ghiaie e le sabbie del fondo, finirebbero col logorarne l'involucro isolante e romperla. Si protegge l'anima fabbricandole attorno una corda di fili di ferro od acciaio zincato; questa corda si chiama armatura. Fra l'anima e l'armatura si frappone una imbottitura di juta tannata. Sull'armatura si dispone anche una fasciatura di tela catramata, destinata a proteggerla dalla ruggine e ad impedire al cavo di strisciare sui tamburi metallici durante i lavori.

In un mare molto profondo le cause esterne dei guasti non sono numerose; laggiù non esistono quei grossi animali che possono addentare un cavo e spezzarlo violentemente, o quei coralli e piante marine che a poco a poco corrodono l'armatura; niente turba la tranquillità di quei fondi, ove la natura sembra sonnecchiare. Qui il cavo deve essere molto leggero, ma molto resistente alla trazione per sopportare il proprio peso durante i lavori. Il tipo comune ha l'armatura di 15 fili d'acciaio da 2,5 $\mathrm{mm}$ di alta resistenza (peso per metro in aria $1 \mathrm{~kg}$, in acqua di mare circa 700 grammi, diametro 
esterno circa $20 \mathrm{~mm}$ ). Per contro, vicino alle coste, queste cause esterne di distruzione si fanno vivamente sentire, e l'armatura deve essere robustissima, formata, per esempio, di 10 fili di ferro da 9,5 $\mathrm{mm}$ (peso per metro $7 \mathrm{~kg}$ circa, diametro esterno 50 $\mathrm{mm}$ circa). Fra questi due tipi estremi è facile immaginare dei tipi intermedi da posare man mano che il fondo va degradando.

La posa di un cavo si fa a questo modo: studiato bene il fondo del mare sulle carte nautiche e magari mediante una apposita campagna preventiva di scandagli, si fissano gli approdi e si segna su dette carte il tracciato da seguire.

La nave si àncora a poca distanza da uno degli approdi e si manda a terra la testa del cavo telegrafico da posare, sorretta da galleggianti perchè non strisci sul fondo. Arrivata a terra, la cima viene introdotta in una trincea sino entro al casotto d'approdo. Poi la nave parte a piccola velocità seguendo il tracciato previsto.

Già sappiamo che alla costa è destinata una certa lunghezza di cavo pesante, per esempio un miglio, sino a raggiungere, poniamo, 10 metri di fondo; passa così questo miglio di cavo, al quale, durante l'imbarco, sono già state attaccate le lunghezze richieste, di cavi intermedi e di fondo. Il cavo che esce dalle vasche va al tamburo della macchina di posa, passa a un dinamometro, e quindi è guidato alla puleggia di poppa, donde scende in mare. Si regola opportunamente la velocità della nave e la frenatura del cavo, in modo da non posarne in grande eccesso, ma abbastanza abbondante (imbando) in modo di permetterne poi il sollevamento in caso di riparazioni. Passano poi il cavo intermedio pesante (supponiamo tre miglia) sino ai fondi di circa 100 metri, l'intermedio leggero sino ai fondi di circa 200 metri (e supponiamo 5 miglia) e quindi il cavo di fondo, che costituirà la parte maggiore della linea. Man mano che si arriva ai cavi più leggeri, la velocità della nave viene aumentata, sino a raggiungere 5-6 miglia all'ora quando si posa il cavo di fondo.

Posata così una certa lunghezza di cavo, esso viene tagliato e la cima immersa in mare ed affidata ad una boa. Perchè questo arresto? Perchè non si potrebbe invero continuare la posa sino a raggiungere l'altro approdo, poichè ad ogni atterraggio spetta una certa lunghezza di cavi di sponda ed intermedi; non si potrebbe regolare lo svolgimento del cavo così bene da raggiungere l'altro approdo coi tipi di cavo ad esso destinati. Si riprende quindi l'operazione nell'identico modo dall'approdo opposto, posando il cavo grosso di sponda, i vari tipi intermedi, e quello di fondo fino a raggiungere la boa; presa allora a bordo la cima boata, si congiunge al cavo nuovamente posato e si getta in mare il giunto, detto giunto finale, che completa la linea. 
Durante la posa si esperimenta in modo continuo il cavo per accertarne le perfette condizioni elettriche e assicurarsi che non si manifesti alcun guasto.

I cavi sono soggetti a guastarsi per varie cause. Quando si manifesta un guasto in un cavo posato, si fanno dai casotti d'approdo esperimenti elettrici per localizzarne la distanza, in miglia, dal casotto; essa viene riportata sulle carte nautiche ove è tracciato il cavo. Non è possibile dare un'idea di questi esperimenti laboriosi e delicati; diremo solo che tale posizione viene determinata con un errore quasi sempre trascurabile.

La nave parte quindi per questo punto, ove mette in mare una boa segnale, punto di riferimento per tutti i lavori. Si sposta quindi due o tre miglia di traverso al tracciato e cala in fondo al mare, da una delle puleggie di prua, un grappino assicurato a una lunga gomena o cavo speciale da grappino; quindi fa lentamente delle piccole bordate attraverso al tracciato del cavo telegrafico. A bordo la gomena del grappino passa dalla puleggia di prua ad un dinamometro e quindi al tamburo della macchina a salpare.

Durante il grappinaggio il dinamometro segna una tensione dovuta al peso della gomena del grappino, alla resistenza che essa incontra nell'acqua ed allo sforzo che il grappino fa per arare il fondo. Quando il cavo telegrafico è afferrato, viene sulle prime un po' trascinato sul fondo, poi, messo in tensione, reagisce lentamente; e a bordo si osserva che il dinamometro sale dolcemente. Se si fosse invece incocciato uno scoglio, la tensione salirebbe bruscamente. Dal graduale aumento di tensione si giudica che il cavo può essere preso. Si ferma allora la nave e si salpa dolcemente il grappino, che porterà a bordo il doppino di cavo telegrafico a cavallo delle marre (in una riparazione del cavo Napoli-Palermo, per sollevare da circa 3700 metri di profondità, impiegammo 18 ore e il dinamometro arrivò a segnare una tensione di 20 tonnellate).

Tagliato il doppino, si sperimentano le condizioni elettriche dei due pezzi del cavo: uno è generalmente buono e si rimette in mare affidandolo ad una boa; l'altro, contenente il guasto, si salpa, sino a che, dalle prove elettriche, si nota che il guasto è venuto a bordo. Si taglia allora il cavo al di là del guasto, lo si giunta col cavo nuovo disponibile a bordo e si posa dirigendo verso la boa, alla quale è stata affidata l'altra cima. Questa si salpa e si giunta col cavo nuovamente posato, calando poi in mare il giunto finale.

Se il cavo fosse invece rotto, la riparazione procede in modo analogo; soltanto si dovranno grappinare le due cime ai due lati della rottura, col grappino d'arresto, che impedisce al cavo afferrato di scivolare durante il salpamento del grappino stesso. 


\section{* L'autore}

Laureatasi a Pisa con una tesi sul primo viaggio di Vasco da Gama in India, è stata borsista della Fundação Calouste Gulbenkian, della Biblioteca Nacional de Portugal, della Comissão Nacional para as Comemorações dos Descobrimentos Portugueses e dello Instituto Camões. È autrice di articoli e saggi accademici, nonché di due opere in volume fra cui Fonti italiane dei secoli $\mathrm{XV}$ XVII sull'espansione portoghese (Pisa, ETS, 2000). Ha partecipato come relatrice a conferenze, seminari, workshop. Giornalista pubblicista, dal 2011 è membro della Research Users Advisory Committee facente capo alla European Holocaust Research Infrastructure.

URL: < http://www.studistorici.com/progett/autori/\#Carnemolla >

\section{Per citare questo articolo:}

CARNEMOLLA, Stefania Elena, «ll mare per comunicare», Diacronie. Studi di Storia Contemporanea : Spazi, percorsi e memorie, 29/10/2013,

URL:<http://www.studistorici.com/2013/10/29/carnemolla_numero_15/ >

Diacronie Studi di Storia Contemporanea www.diacronie.it

Risorsa digitale indipendente a carattere storiografico. Uscita trimestrale.

redazione.diacronie@hotmail.it

Comitato di redazione: Marco Abram - Jacopo Bassi - Luca Bufarale - Alessandro Cattunar - Elisa Grandi - Deborah Paci - Fausto Pietrancosta - Matteo Tomasoni - Luca Zuccolo

Diritti: gli articoli di Diacronie. Studi di Storia Contemporanea sono pubblicati sotto licenza Creative Commons 2.5.

Possono essere riprodotti a patto di non modificarne i contenuti e di non usarli per fini commerciali. La citazione di

estratti è comunque sempre autorizzata, nei limiti previsti dalla legge. 